\title{
Global survey of star clusters in the Milky Way: The open cluster distance scale
}

\author{
N. V. Kharchenko, ${ }^{1,2,3}$ A. E. Piskunov,${ }^{2,3,4}$ S. Röser, ${ }^{2}$ E. Schilbach, ${ }^{2}$ \\ and R.-D. Scholz ${ }^{3}$ \\ ${ }^{1}$ Main Astronomical Observatory, 27 Academica Zabolotnogo Str., 03680 Kiev, Ukraine \\ email: nkhar@mao.kiev.ua \\ ${ }^{2}$ Astronomisches Rechen-Institut, Zentrum für Astronomie der Universität Heidelberg, \\ Mönchhofstr. 12-14, 69120 Heidelberg, Germany \\ email: roeser@ari.uni-heidelberg.de, elena@ari.uni-heidelberg.de \\ ${ }^{3}$ Leibniz Institut für Astrophysik Potsdam, An der Sternwarte 16, 14482 Potsdam, Germany \\ email: rdscholz@aip.de \\ ${ }^{4}$ Institute of Astronomy of the Russian Academy of Sciences, 48 Pyatnitskaya Str., \\ 109017 Moscow, Russia \\ email: piskunov@inasan.ru
}

\begin{abstract}
We present the Milky Way Star Clusters (MWSC) survey based on 470 million stars extracted from the all-sky catalogues PPMXL and 2MASS. The target list comprises 3784 entries and includes all open and globular clusters, as well as all cluster candidates, known at present. By means of a combined photometric/astrometric pipeline process, we determine individual membership probabilities for cluster stars and provide fundamental cluster parameters such as cluster centre coordinates, mean proper motions, radial velocity, distance, reddening, age and tidal radius. This sample includes $\sim 700$ clusters with fundamental parameters based on optical data and offers a good opportunity for a comparison of the near-infrared (NIR) and optical cluster distance scales. We find that distances and reddening values obtained from the colourmagnitude diagrams (CMDs) in the NIR agree well with earlier distance estimates based on optical CMDs. These newly obtained homogeneous distances, together with the other cluster parameters, allow us to study the properties of the open cluster population in the Galactic disk.
\end{abstract}

Keywords. open clusters and associations: general, globular clusters: general, Galaxy: fundamental parameters, Galaxy: structure, Galaxy: evolution

\section{Introduction}

Star clusters are important representatives of the Galactic population. Their significance is based on the accuracy with which their fundamental parameters (e.g., distances, ages) can be determined, which is considerably higher for clusters than for stars. For a long time, the efforts of astronomers concentrated on studying individual clusters. However, with new all-sky surveys completed during recent decades, there are now opportunities to study large samples of clusters, thus providing clues for our understanding of the history of star formation and evolution of Galactic populations on large spatial scales.

Our current project, 'Milky Way Star Clusters' (MWSC), is based on exploitation of the 2MAst catalogue (2MASS with Astrometric data). We aim at identifying all known cluster-like objects (open and globular clusters and candidates, compact associations, moving groups, remnant clusters, etc.) and determining their fundamental parameters. In the framework of this project, we significantly increased the number of clusters with homogeneous sets of cluster parameters. For example, the limiting distance of the sample 
Table 1. Comparison of COCD and MWSC surveys.

\begin{tabular}{lll}
\hline & COCD & MWSC \\
\hline Basic castalogue & Hipparcos+Tycho & PPMXL \\
Working catalogue & ASCC-2.5 & 2MAst \\
Number of stars & $2.5 \mathrm{mln}$. & $470 \mathrm{mln}$. \\
Limiting magnitude & $V=12.5 \mathrm{mag}$ & $K_{\mathrm{s}}=15.3 \mathrm{mag}$ \\
Basic stellar data & $\mathrm{RA}, \mathrm{Dec}, \mathrm{PM}, B V$ & $\mathrm{RA}, \mathrm{Dec}, \mathrm{PM}, J H K_{\mathrm{s}}$ \\
Additional stellar data & $J H K_{\mathrm{s}}, \mathrm{Sp}, \mathrm{RV}$ & $B V, \mathrm{Sp}, \mathrm{RV}$ \\
Number of clusters & 650 & 3006 \\
Limiting distance & $\sim 8 \mathrm{kpc}$ & $>20 \mathrm{kpc}$ \\
Sample completeness & $0.85 \mathrm{kpc}$ & $2 \mathrm{kpc}$ \\
\hline
\end{tabular}

now extends to a few tens of $\mathrm{kpc}$, and the completeness limit of $\sim 2 \mathrm{kpc}$ reaches the nearby spiral arms. Here we present the distance scale derived for the Milky Way star clusters and give a few examples of their spatial distribution.

\section{The Milky Way Star Cluster Survey}

The MWSC survey exceeds the optical survey of Galactic star clusters COCD (Kharchenko et al. 2005a,b) by almost a factor of five (see Table 1). Our basic data were extracted from the all-sky catalogue PPMXL (Röser et al. 2010), which contains positions and proper motions in the ICRS (international celestial reference system) and low-accuracy photometry from the USNO-B catalogue (Monet et al. 2003) for some 900 million objects (down to $V \approx 20 \mathrm{mag}$ ). For a subset of about 470 million entries, accurate $J, H, K_{s}$ magnitudes are available from 2MAss. This subset, 2MAst, was used to verify the cluster nature and to determine cluster parameters in homogeneous photometric and astrometric all-sky systems.

Using the technique described by Kharchenko et al. (2012), we examined all cluster-like objects found in the literature. From the target list of 3784 objects, we confirmed 3006 open, globular clusters and compact associations, and determined their astrophysical parameters. The rejected objects turned out to be random clusterings of field stars, were too faint for a proper identification in 2MAst, or were simply duplicate entries.
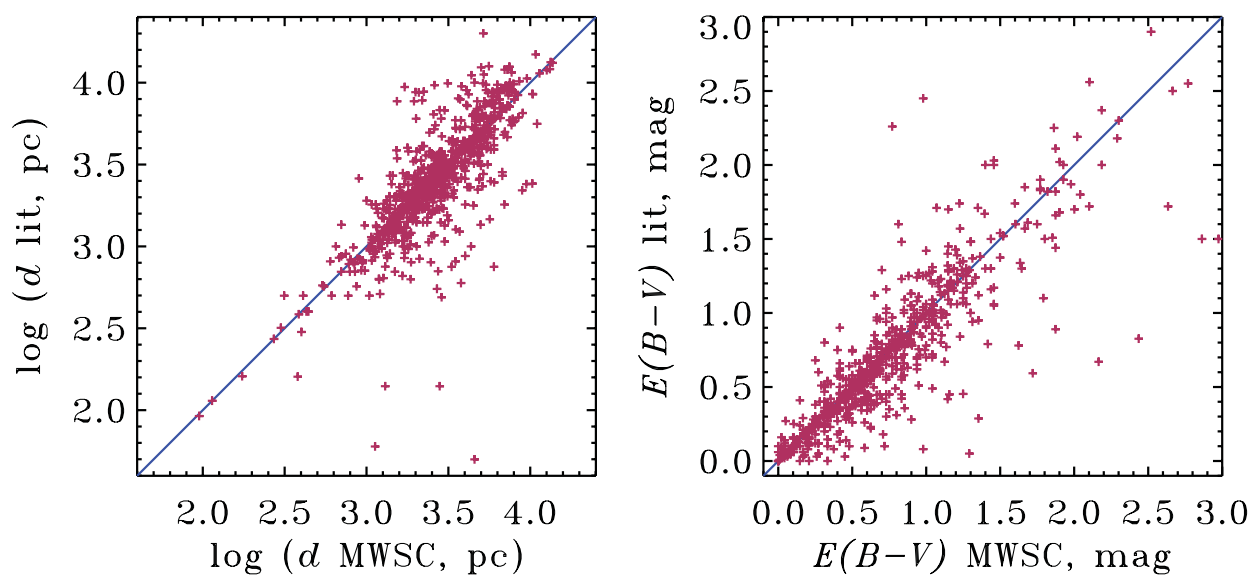

Figure 1. Comparison of derived cluster distances (left) and reddening values (right) with the corresponding data from the literature. 


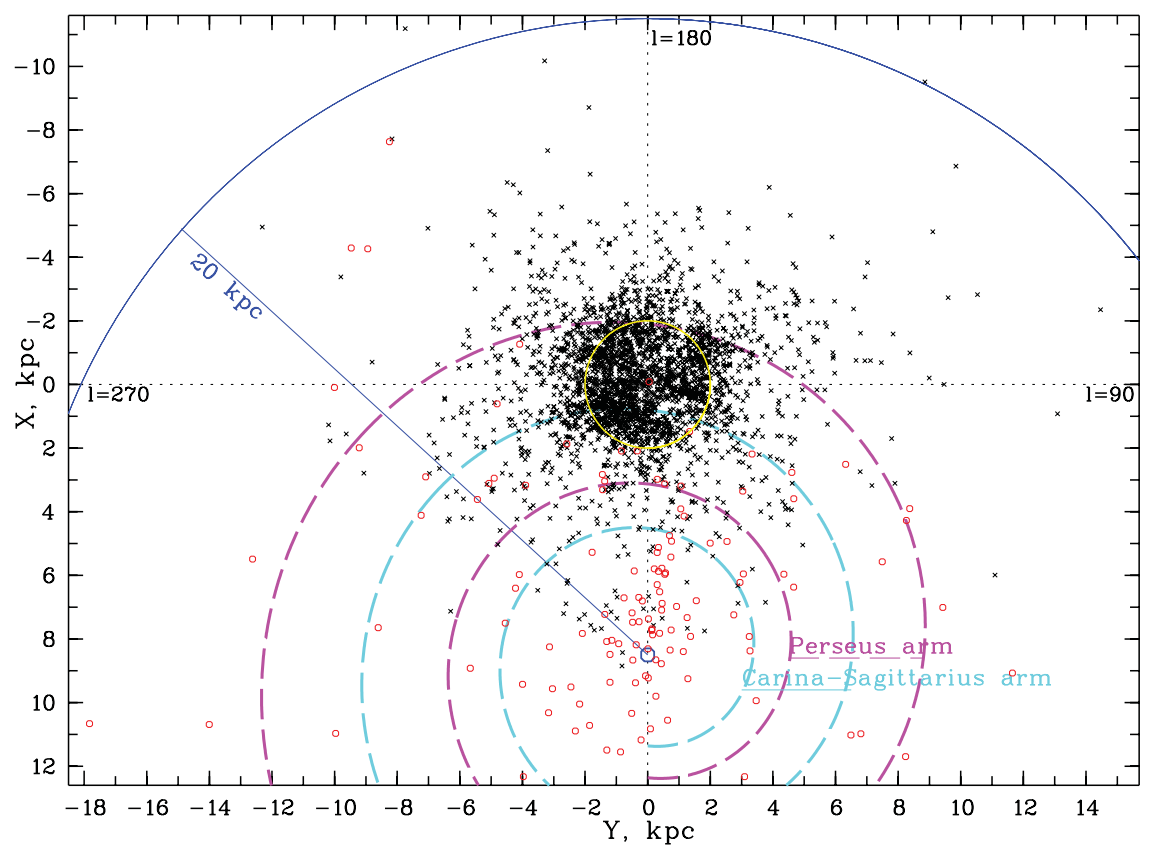

Figure 2. Distribution of star clusters projected onto the Galactic $X Y$ plane, centred on the location of the Sun. Dashed curves are provisional positions of Perseus (dark), and Carina-Sagittarius (light) arms. The light circle around the Sun indicates the completeness area of the MWSC. Crosses and open circles mark open and globular clusters, respectively.

\section{Cluster distances}

In general, the parameter determination follows the rules applied previously to the COCD clusters (Kharchenko et al. 2005a). Separation of cluster members from the field is based on kinematic, photometric and spatial criteria. The cluster parameters were determined iteratively, with the initial values taken either from the literature or based on visual estimates.

Determination of the distance moduli and reddening values is done by fitting theoretical isochrones to the observed $K_{\mathrm{s}}-(J-H), K_{\mathrm{s}}-\left(J-K_{\mathrm{s}}\right),\left(H-K_{\mathrm{s}}\right)-(J-H)$ and $Q\left(J H K_{\mathrm{s}}\right)-$ $\left(J-K_{\mathrm{s}}\right)$ diagrams. We used Padova isochrones (Marigo et al. 2008) combined with pre-main-sequence tracks from Siess et al. (2000), which we converted to the 2MASS photometric system. The choice of a proper isochrone presumes knowledge of the age of a cluster. This means that the cluster reddening, distance modulus and age are determined in an iterative process.

In Fig. 1 we compare our results with cluster parameters from the literature, mainly from Loktin et al. (2001), whose determinations were based on photoelectric $U B V$ photometry and represent the most extended homogeneous set of cluster parameters based on optical photometry. We conclude that our near-infrared distances and colour excesses agree well with the optical data: there is no bias, neither in distance (from $100 \mathrm{pc}$ to 10 kpc) nor in $E(B-V)$ (over $\sim 3$ mag in reddening).

\section{Spatial distribution of the cluster population}

In Fig. 2 we show the distribution of open clusters in the Galactic $X Y$ plane. The clusters cover a large range of galactocentric distances, from the central regions of the 

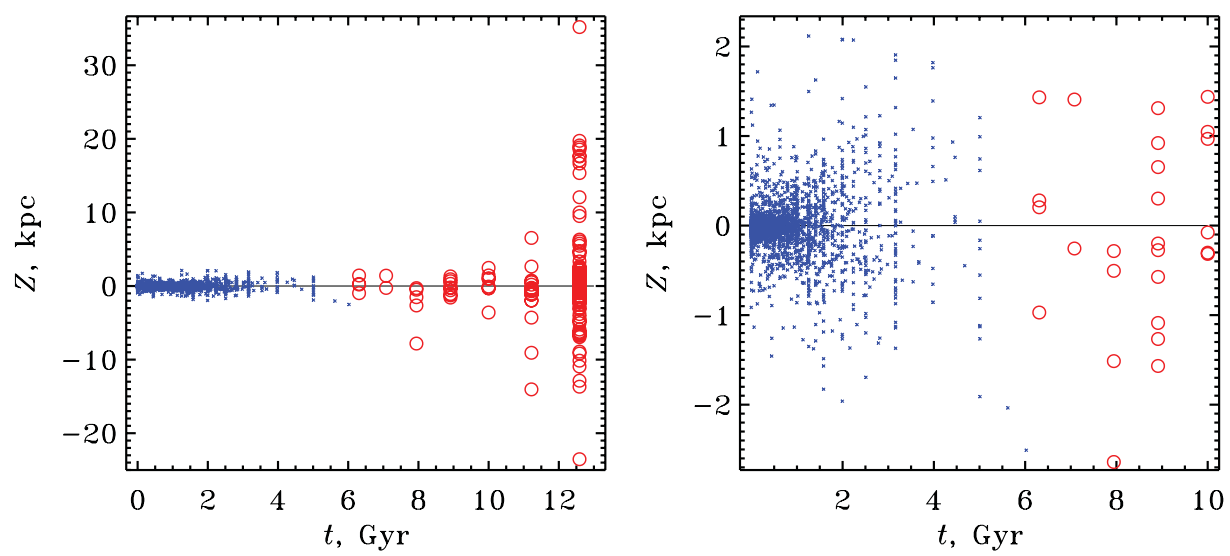

Figure 3. $Z$ coordinate-age relation for the clusters of interest. Open and globular clusters are shown as small crosses and open circles, respectively. The right-hand panel shows the relation for younger ages in more detail.

Galactic disk to the outskirts at $15-20 \mathrm{kpc}$ from the Galactic Centre. This large sample of clusters with known distances offers a possibility to study the spatial distribution of different cluster groups selected by their age, kinematics, etc.

In Fig. 3 we show the $Z$ distance of star clusters from the Galactic plane as a function of cluster age. The strong concentration of open clusters close to the Galactic plane stops abruptly at a cluster age of approximately 1-2 Gyr. The scale height of open clusters increases with age and shows a continuous transition to the distribution of the globular clusters.

\section{Acknowledgements}

This work is the result of a German-Russian collaboration supported by DFG grant RO528/10-1, and by SFB 881, 'The Milky Way System' (subproject B5). Part of this work was supported by RFBR grant 10-02-91338.

\section{References}

Kharchenko, N. V., Piskunov, A. E., Röser, S., Schilbach, E., \& Scholz, R.-D. 2005, A\&A, 438, 1163

Kharchenko, N. V., Piskunov, A. E., Röser, S., Schilbach, E., \& Scholz, R.-D. 2005, A\&A, 440, 403

Kharchenko, N. V., Piskunov, A. E., Schilbach, E., Röser, S., \& Scholz, R.-D. 2012, A\&A, 543, A156

Loktin, A. V., Gerasimenko, T. P., \& Malysheva, L. K. 2001, Astron. Astrophys. Trans., 20, 607

Marigo, P., Girardi, L., Bressan, A., Groenewegen, M. A. T., Silva, L., \& Granato, G. L. 2008, $A \mathscr{E} A, 482,482,883$

Röser, S., Demleitner, M., \& Schilbach, E. 2010, AJ, 139, 2440

Monet, D. G., Levine, S. E., Canzian, B., et al. 2003, AJ, 125, 984

Siess, L., Dufour, E., \& Forestini, M. 2000, A\&A, 358, 593 